American J. of Engineering and Applied Sciences 2 (4): 590-602, 2009

ISSN 1941-7020

(C) 2009 Science Publications

\title{
Combined Thermal Radiation and Mixed Convection in an Inclined Circular Duct
}

\author{
${ }^{1}$ W. Lakhal, ${ }^{2}$ S. Trabelsi, ${ }^{2}$ E. Sediki and ${ }^{1}$ M. Moussa \\ ${ }^{1}$ Laboratory of Modeling in Hydraulics and Environment, \\ National Engineering School of Tunis, BP 37, 2092 Tunis, Tunisia \\ ${ }^{2}$ Radiative Transfer Laboratory, Department of Physics, \\ Faculty of Sciences of Tunis, 2092 Tunis, Tunisia
}

\begin{abstract}
Problem statement: Modeling thermal radiation with simultaneous buoyancy and forced convection for real gases flowing inside system with complex geometry is a difficult task encountered in engineering applications. Purpose of this study was to research numerically the interaction of mixed convection and thermal radiation in laminar air flow inside an inclined cylindrical duct with Uniform Wall Heat Flux (UWHF). This study highlighted the radiative double effects of water vapor in air flow on thermal and on dynamic fields. Approach: Flow equations and energy balance equation were solved simultaneously with temperature dependant thermophysical properties. An implicit finite difference technique was used to solve mass, momentum and energy equations. In order to take into account the non-gray radiative behavior of water vapor $\left(\mathrm{H}_{2} \mathrm{O}\right)$, a global absorption distribution function model was used to represent the infrared radiative properties. Results: Results were presented in term of temperature, velocity and radiative power fields and of evolution of bulk temperature and Nusselt numbers. Effects of thermal radiation on temperature and on velocity distributions were also examined. Conclusion/Recommendations: It was shown that inclination angle of duct had a significant effect on thermal and dynamic fields especially for thick medium. Radiation strongly affected the velocity profiles. Numerical results were discussed referring to available experimental data in order to improve estimations of engineering parameters.
\end{abstract}

Key words: Inclined duct, inclination angle, mixed convection, thermal radiation, cylindrical duct

\section{INTRODUCTION}

During the past few decades, an increased interest has been drawn to further enhance the understanding of spectrally thermal radiation effects on temperature and velocity fields for laminar flow in horizontal, vertical or inclined cylindrical ducts. Many transport processes exist in industrial applications in which the heat transfer occurs simultaneously as a result of combined thermal radiation and buoyancy effects.

The interactions of mixed convection and radiation are described using some approximations and assumptions that generally do not deal with reality. These hypotheses give a small accuracy to engineering analysis due to the complicated solution method of transport equations when radiative behavior of real gas is taken into account. As pointed out by many researchers ${ }^{[1-3]}$, the main fundamental difficulties in the problem of combined radiative and convective heat transfer are the non-gray behavior of gases, the multidimensional nature of radiative transfer and the resulting coupling between different volume elements in the medium.

Laminar convection of non radiating gas in horizontal, vertical or inclined cylindrical tubes has been extensively studied. Much studies, both theoretical and experimental were made. Many authors examined buoyancy effects on thermal and dynamic fields inside vertical or inclined ducts for two thermal boundary conditions. Uniform Wall Temperature (UWT) and Uniform Wall Heat Flux (UWHF) are of a particular interest although in general the industrial applications are in an intermediate regime.

Concerning laminar mixed convection heat transfer without radiation for air flowing inside tubes under UWT boundary condition, theoretical and experimental studies are carried out by ${ }^{[4,5]}$. Other references in this field may be found in classical text books or review studies $^{[6,7]}$.

Under condition of UWHF, some recent experiments have been conducted by Mohammed and

Corresponding Author: E. Sediki, Radiative Transfer Laboratory, Department of Physics, Faculty of Sciences of Tunis, 2092 Tunis, Tunisia 
Mohammed and Salman to investigate the mixed convection heat transfer in circular horizontal duct ${ }^{[8-11]}$, vertical duct ${ }^{[12-15]}$ and inclined duct ${ }^{[16]}$. Results show that a decrease of the inclination angle induces an increase on the Nusselt number values. The average Nusselt numbers have been correlated with the Rayleigh numbers and Reynolds numbers in empirical correlations. The average heat transfer results and Nusselt numbers were correlated with empirical correlations in terms of dependent parameters of Grashof, Prandtl and Reynolds numbers.

Concerning the interaction between radiative and convective heat transfer for laminar flow, studies have been conducted numerically and experimentally for the two boundary conditions. Seo et al. ${ }^{[17]}$ have considered a laminar flow of mixture of carbon dioxide, water vapor and nitrogen inside a circular tube with UWT. They concluded that the convective heat fluxes in combined heat transfer are greater than heat fluxes for pure convection. The heat transfer characteristics of non gray gas mixture strongly depend on the molar fraction of gases. In fact, a small amount of radiating gases can change the heat transfer significantly. Sediki et al. ${ }^{[2,3]}$ investigated the interaction between the 2-D thermal radiation and convection for laminar absorbing-emitting real gases flowing inside circular duct. The radiative properties of the flowing gases were modeled by using the global Absorption Distribution Function model (ADF). They conclude that the effects of the radiative axial component remain noticeable as long as the difference between wall and fluid bulk temperatures is significant.

Recently, Selçuk et al. ${ }^{[18]}$ studied the laminar non isothermal flow of a radiatively participating medium along an axisymetric circular duct where the wall temperature undergoes a sudden step change at some position along the tube. They proposed a numerical solution of time dependent Navier-Stockes equations with Radiative Transfer Equation (RTE) in 2-D cylindrical geometries. Lately, Chiu and $\mathrm{Yan}^{[19]}$ investigated the radiation effect on the characteristics of the mixed convection fluid flow and heat transfer in inclined rectangular ducts. Results reveal that radiation effects have a considerable impact on the heat transfer and tend to reduce the thermal buoyancy effects. The development of the bulk temperature is enhanced by the radiation effects.

Many others studies of combined heat transfer in ducts subjected to UWHF have been performed to illustrate the interaction between mixed convection and thermal radiation. Earlier study was made by Greif ${ }^{[20]}$. Theoretical and experimental results have been carried out for laminar convection with radiation of carbon dioxide. Results show that the effect of radiation to decrease temperature differences reduces the influence of natural convection. Smith and Clausen ${ }^{[21]}$ treated the problem for engineering applications by considering an emitting-absorbing mixture of carbon dioxide and water vapor inside circular tube. The importance of including the radiative term in energy balance has been exposed.

Study of Kim and Smith ${ }^{[22]}$ has looked at the interaction of radiative and conductive heat transfer for a radiatively participating real gas stagnant in a cylindrical circular enclosure. They found that the effect of radiation on wall heat fluxes is important compared to conduction effect. A description of these coupled heat transfer effects in vertical tube for a gray gas was also presented by Yang $^{[23,24]}$. It was shown that the local Nusselt number is enhanced with the increase of radiation-conduction parameter.

In order to draw near the reality and to give compatible results in many combustion applications, it is important to take into account the non-gray behavior of gas when modeling the radiative transfer. These reasons motivate this study.

We consider the flow of gaseous mixtures of $\left(\mathrm{H}_{2} \mathrm{O}-\mathrm{N}_{2}\right)$ inside a circular inclined duct. The combined effects of convective and radiative heat transfer on the temperature and velocity distributions and on the Nusselt number variation for an absorbingemitting non-gray gas flow are examined. Thus, the main aim of the present study is to analyze the effects of inclination angle of duct subjected to UWHF on combined convection and two dimensional thermal radiation problems for real gases.

We highlight the double effects of thermal radiation, related to the presence of water vapor in the flow, on combined heat transfer. The direct effect is due to the fact that the temperature distribution in the fluid determines the local radiative flux through the action at distance. The indirect effect is on the velocity field through the temperature dependent fluid properties. The radiative heat flux alters the temperature distribution in the duct and modifies the convective flux and hydrodynamic fields.

The radiative properties of the non-gray gas are modeled by using the Absorption Distribution Function (ADF) infrared radiative property model where the spectral correlation phenomenon is taken into account.

The effects of inclination angle on the wall temperature, on the bulk temperature and on the Nusselt numbers distributions are studied. In the last part of this study, experimental results of Mohammed and Salman ${ }^{[16]}$ are discussed referring to numerical ones in order to give a first order of engineering parameters 
estimations for combined radiation and convection heat transfer problem.

\section{MATERIALS AND METHODS}

Solution of combined radiative and convective heat transfer: The investigated situation corresponds to a laminar steady flow of heated non-scattering gas mixtures inside an inclined circular duct. Figure 1 show a schematic view of the system. The gas inlet temperature is assumed to be uniform and indicated by $\mathrm{T}_{0}$. The internal flow is fully developed and the velocity profile is parabolic.

In the formulation of the heat transfer phenomena without radiation all the usual boundary layer approximations were made. Furthermore viscous dissipation is neglected.

We restrict our considerations to axially symmetric heated flows. The mass, momentum and energy balance equations are given respectively by:

$\nabla \cdot(\rho \overrightarrow{\mathrm{V}})=0$

$\nabla \cdot(\rho \overrightarrow{\mathrm{V}} \overrightarrow{\mathrm{V}})=-\nabla p+\nabla \cdot \nabla(\mu \overrightarrow{\mathrm{V}})+\rho \overrightarrow{\mathrm{g}}$

$\nabla \cdot(\rho \mathrm{h})=-\nabla \cdot \overrightarrow{\mathrm{q}}_{\mathrm{cd}}-\nabla \cdot \overrightarrow{\mathrm{q}}_{\mathrm{rad}}$

Where:

$\overrightarrow{\mathrm{V}} \quad=$ The velocity vector $\mathrm{h}(\mathrm{T})=\int^{\mathrm{T}} \mathrm{C}_{\mathrm{p}}(\mathrm{T}) \mathrm{dT}$

$\rho$ and $\mu=$ Designate respectively the enthalpy per unit mass, the temperature-dependent density and the viscosity

$\overrightarrow{\mathrm{q}}_{\mathrm{rad}}$ and $\overrightarrow{\mathrm{q}}_{\mathrm{cd}}=$ Respectively the radiative vector flux and the conductive vector flux

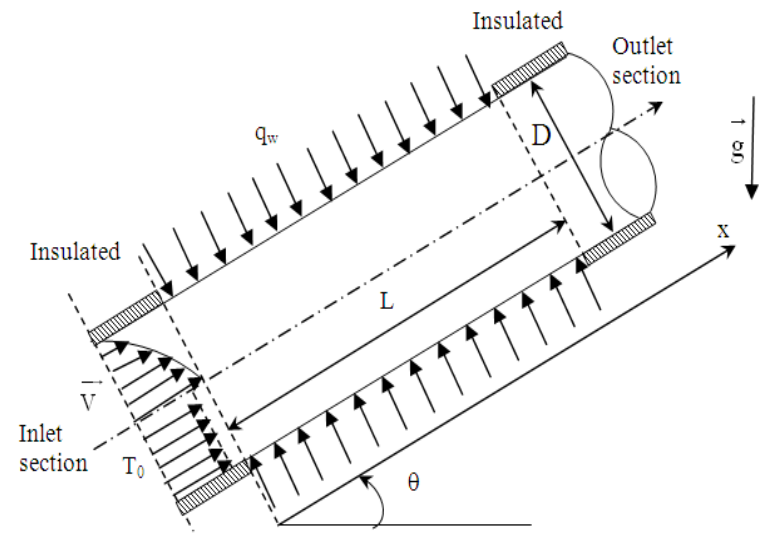

Fig. 1: Geometrical and physical conditions
The boundary conditions for the considered problem are summarized as follow:

- The no-slip condition at the wall and the impermeability of the wall:

$$
\mathrm{u}(\mathrm{x}, \mathrm{R})=0 \text { and } \mathrm{v}(\mathrm{x}, \mathrm{R})=0
$$

- The subjected uniform wall heat flux gives the following conditions:

$\mathrm{q}_{\mathrm{w}}=\left.\frac{\lambda}{\mathrm{C}}\left(\frac{\partial \mathrm{h}}{\partial \mathrm{r}}\right)\right|_{(\mathrm{x}, \mathrm{R})}$ and $\left.\frac{\partial \mathrm{h}}{\partial \mathrm{r}}\right|_{(\mathrm{x}, 0)}=0$

- The radiative boundary condition is given by:

$I_{j}(\vec{u})=\varepsilon_{w} a_{j}\left(T_{w}\right) \frac{\sigma T^{4}}{\pi}+\frac{\left(1-\varepsilon_{w}\right)}{\pi} \int_{\vec{n} \dot{u}<0} I_{j}\left(\vec{u}^{\prime}\right)|\vec{n} \quad \vec{u}| d \Omega$

where, $\varepsilon_{w}, a_{j}, \vec{n}$, $\vec{u}$ and $\overrightarrow{\mathrm{u}}{ }^{\prime}=$ The emissivity of the wall, the weights as a parameter of ADF radiative property model, the direction normal to the boundary, the direction of the emission and the direction of incoming radiation respectively:

- For $\mathrm{x}=0$ we have the following inlet conditions:

$\mathrm{v}(0, \mathrm{r})=0, \mathrm{u}(0, \mathrm{r})=2 \mathrm{u}_{0}\left(1-\left(\frac{\mathrm{r}}{\mathrm{R}}\right)^{2}\right)$

where, $\mathrm{u}_{0}$ is the average velocity at the entrance:

$\mathrm{h}(0, \mathrm{r})=\mathrm{h}\left(\mathrm{T}_{0}\right)$

The flow rate is assumed to be fixed by a metering device

Since the system of equations formulated above is assumed parabolic in $\mathrm{x}$ direction, no flow boundary conditions are required at the outlet section of the computational domain. The significant temperature difference imposed between the walls and the gas requires the use of temperature-dependent fluid properties.

The gas is assumed to obey the perfect gas law and the thermophysical properties are approximated by temperature dependent functions generated from the data given in ${ }^{[27]}$ for pure gases. For mixtures, we use the Buddenberg-Wilke approximation for $\mu$ and the Mason-Saxena approximation for the thermal conductivity $^{[27]}$. 
The total radiative intensity I ( $\vec{u}, s)$ at curvilinear abscissa $\mathbf{s}$ and for the direction $\overrightarrow{\mathrm{u}}$ is written as the sum of the partial intensities $I_{j}(\vec{u}, s)$ which satisfies the radiative transfer equation:

$$
\frac{\partial \mathrm{I}_{\mathrm{j}}(\overrightarrow{\mathrm{u}}, \mathrm{s})}{\partial \mathrm{s}}=\sum_{\mathrm{j}=1}^{\mathrm{N}} \mathrm{k}_{\mathrm{j}}(\mathrm{s}) \mathrm{X}_{\mathrm{H}_{2} \mathrm{O}} \mathrm{p}(\mathrm{s})\left[\mathrm{a}_{\mathrm{j}}(\mathrm{s}) \frac{\sigma \mathrm{T}^{4}}{\pi}-\mathrm{I}_{\mathrm{j}}(\overrightarrow{\mathrm{u}}, \mathrm{s})\right]
$$

The divergence of the radiative flux or the radiative source term, appearing in the energy equation, is given by:

$$
\nabla \overrightarrow{\mathrm{q}_{\mathrm{rad}}}=\sum_{\mathrm{j}=1}^{\mathrm{N}} \mathrm{k}_{\mathrm{j}}(\mathrm{s}) \mathrm{X}_{\mathrm{H}_{2} \mathrm{O}} \mathrm{p}(\mathrm{s}) \int_{4 \pi}\left[\mathrm{a}_{\mathrm{j}}(\mathrm{s}) \frac{\sigma \mathrm{T}^{4}}{\pi}-\mathrm{I}_{\mathrm{j}}(\overrightarrow{\mathrm{u}}, \mathrm{s})\right] \mathrm{d} \Omega
$$

The discrete-direction method is adapted to ADF model $^{[25,26]}$, to solve the radiative transfer problem without any further approximations. In one hand, the directional integration quadrature is used for a regular integration with prescribed angular increments and solid angles. In the other hand intensity interpolations are used instead of integrations over control volumes.

Numerical procedure: If the 2-D radiation calculations are used, the system of equations corresponding to mass, momentum and energy balance is inherently elliptic. The origin of this ellipticity is the radiative term due to its action at distance. So, in the case of parabolic flow the marching solution procedure in the axial direction cannot be applied without the knowledge of the radiative source term at each point of the system.

In this study, we propose, to use a numerical method able to transform the elliptic problem to a series of parabolic ones where we can use a marching solution from the inlet section $(x=0)$ of the duct until the outlet section $(\mathrm{x}=\mathrm{L})$. The use of a coupled radiative calculation code with a flow field computational code is the basic idea to solve simultaneously the coupled problem.

Three mesh structures are used to solve numerically the combined convective and radiative problem: the 1D radiative grid, the $2 \mathrm{D}$-radiative grid and the convective grid. The radiative heat transfer solution code needs a grid that is coarser than the grid used in the CFD code for solving mass and momentum balance equations. The temperature field, needed for calculating the radiative source term and for the thermophysical properties, is given by the CFD code used in the flow calculation. After calculating the volumetric radiative power in the coarse mesh structure, source terms are interpolated or extrapolated over the fine mesh structure and added to the overall energy balance equation.
The method starts with the prediction of the temperature and velocity fields using local 1-D radiative transfer calculations and a marching procedure along the axial direction is used from the inlet section to the outlet section. Thus the radiative source term or the volumetric radiative power dissipated in the medium for a given volume element at the location $x_{i}$ is calculated from a one-dimensional temperature field. In practice, for $1 \mathrm{D}$ radiative transfer calculations, the same numerical procedure is used with six discretized axial sections and a very large spacing between these sections in comparison with the tube diameter.

In a second step, an iterative procedure is undertaken. Two-dimensional radiative powers and fluxes are calculated from the 2-D temperature field and then the flow and energy equations are solved using the marching procedure. The required number of global iterations depends on the optical thickness of the medium.

A constant mesh size is used for the radial direction and a variable one in the axial direction. This mesh size increases far from the entrance, which allows a higher accuracy for the temperature and velocity fields in the thermal entrance region where significant steep axial gradients occur. Typically, we use 160 radial points and 20000 axial ones to compute the flow field. Such refined grid is not required for 2-D radiation calculations; numerical tests show that typically $40 \times 100$ nodes are sufficient for the above configuration. Numerical linear interpolations and extrapolations are used to convert the results from the convective grid mesh to the radiative one and vice versa.

Details on the numerical solution and radiative treatment are available in reference ${ }^{[2,3,28]}$. In fact, when radiative transfer problem is solved, the source terms $\left(\nabla \overrightarrow{\mathrm{q}}_{\text {rad }}\right)$ are known at each point of the medium, then the flow equations become parabolic, an implicit finite marching procedure, similar to that of Patankar and Spalding ${ }^{[29]}$ is used.

At each flow cross section, discretization of momentum and energy equations leads to two tridiagonal matrix inversions. The value of the pressure drop is calculated at each step in such a manner that the velocity field satisfies the integrated continuity equation. This procedure is similar to that used for non-radiating laminar fluid flow in Refs ${ }^{[30,31]}$. The convection part of this parabolic procedure was validated by comparison with the results of ${ }^{[30,31]}$ for laminar forced convection in circular duct. The relative difference, in terms of Nusselt numbers, between our results and the previous investigations do not exceed 3\%. 
However, neglecting the contributions of axial diffusion of momentum and energy in the system of equations is the key of the parabolic boundary-layer approximations for flows considered in this study. Worsoe-Schmidt and Leppert ${ }^{[31]}$ concluded that, for developing flows in circular ducts, the boundary-layer model is valid for $\mathrm{x} /(\mathrm{R} . \mathrm{Re} . \mathrm{Pr})>10^{-3}$. The range of validity of this assumption has been also extensively discussed by Aung and Worku ${ }^{[32,33]}$ and Jeng et al. ${ }^{[5]}$ among others.

Several calculations have been carried out. Dynamic, thermal and radiative fields are computed at each point of the medium. The velocity, temperature and volumetric radiative power 2-D distributions obtained cannot be presented here in full, nor are they easy to use for engineering purposes. The parameters of interest to engineers are: bulk temperature, Nusselt number and heat transfer coefficients.

In the study, the mean bulk temperature is defined by:

$$
\mathrm{T}_{\mathrm{b}}(\mathrm{x})=\frac{\int \rho \mathrm{uh}(\mathrm{x}, \mathrm{r}) 2 \pi \mathrm{rdr}}{\int \rho \mathrm{uC} \mathrm{C}_{\mathrm{p}} 2 \pi \mathrm{rdr}}
$$

The energy transport from gas flow to duct in the presence of thermal radiation depends on two related factors: The fluid temperature gradient at the wall and the rate of radiative heat exchange. So, the overall heat flux at the wall is a sum of the conductive heat flux and the radiative heat flux:

$$
\phi_{\mathrm{tt}}(\mathrm{x})=\phi_{\mathrm{cd}}(\mathrm{x})+\phi_{\mathrm{rad}}(\mathrm{x})
$$

where, $\quad \phi_{\text {rad }}(x)=\frac{\int_{S} \operatorname{div} \overrightarrow{\mathrm{q}}_{\mathrm{rad}} \mathrm{d} S}{2 \pi \mathrm{r}}$ and $\operatorname{div} \overrightarrow{\mathrm{q}}_{\mathrm{rad}}$ is given by Eq. 8 .

The local Nusselt number is defined at the length $\mathrm{x}$ from the entrance as the sum of convective and radiative contributions:

$$
\mathrm{Nu}_{\mathrm{tt}}(\mathrm{x})=\mathrm{Nu}_{\mathrm{cd}}(\mathrm{x})+\mathrm{Nu}_{\mathrm{rad}}(\mathrm{x})
$$

With:

$$
\mathrm{Nu}_{\mathrm{cd}}(\mathrm{x})=\frac{\mathrm{D} \mathrm{q}_{\mathrm{w}}}{\lambda\left(\mathrm{T}_{\mathrm{W}}(\mathrm{x})-\mathrm{T}_{\mathrm{b}}(\mathrm{x})\right)}
$$

and

$$
\mathrm{Nu}_{\mathrm{rad}}(\mathrm{x})=\frac{\mathrm{D} \phi_{\mathrm{rad}}(\mathrm{x})}{\lambda\left(\mathrm{T}_{\mathrm{W}}(\mathrm{x})-\mathrm{T}_{\mathrm{b}}(\mathrm{x})\right)}
$$

Results obtained with various physical parameters (Reynolds number, diameter of duct, gas mixture composition, wall and gas inlet temperature) cannot be put in general correlation formula since gas thermophysical properties strongly depend on the temperature and radiative transfer depends in a highly non-linear manner on length scales and temperature levels. Nusselt number and bulk gas temperature will be presented as first order estimation and can be useful for engineering applications.

\section{RESULTS}

In this numerical study, the emphasis has been placed on solutions for the interaction between mixed convection and radiation in an inclined tube subjected to uniform heat flux at the wall. Various combinations of Reynolds number, diameter of duct, gas mixture compositions and duct inclination angle have been considered.

The objectives of this study is to point up the effect of the water vapor radiative behavior and the effect of the inclination angle on the evolution of temperature, velocity, Nusselt number, bulk temperature and wall temperature.

The governing parameters used in this study are $\mathrm{Re}$ $=400-1500, \mathrm{~T}_{0}=400 \mathrm{~K}$, the cylindrical diameter $\mathrm{D}=$ $0.03-0.1 \mathrm{~m}$, the length of the duct is $\mathrm{L}=0.9 \mathrm{~m}$ and the inclination angle $\theta=0^{\circ}, 30^{\circ}, 60^{\circ}$ and $90^{\circ}$. We consider a various water vapor molar fractions $\left(\mathrm{X}_{\mathrm{H} 2 \mathrm{O}}\right.$ from $\left.0.2-1\right)$ in order to evaluate the magnitude of gas thermal radiation on heat transfer. The duct wall was assumed to be black and the physical property variations of fluid with temperature were accounted for. The wall is heated with uniform heat flux from section $\mathrm{x}=0$ to section $\mathrm{x}=\mathrm{L}$ and insulated section up to $\mathrm{x}=\mathrm{L}$.

In order to discuss agreement or disagreement of numerical and experiments results, an example of calculus domain $(\mathrm{Re}, \mathrm{L}, \mathrm{D}, .$.$) chosen at the last of this$ study is taken identical to that considered by Mohammed and Salman ${ }^{[16]}$.

\section{DISCUSSION}

Combined gas radiation and buoyancy effects on thermal and dynamic fields in an inclined duct: Several numerical calculations have been done for pure laminar convection as well as for combined radiation and laminar convection.

In first time and in order to study the effect of thermal radiation on thermal and dynamic fields, some parameters are considered constant: Molar fraction of water vapor $\mathrm{X}_{\mathrm{H}_{2} \mathrm{O}}=0.6$, duct inclination angle $\theta=60^{\circ}$ 
and different cylindrical diameter $(D=0.03$ or $\mathrm{D}=0.1 \mathrm{~m})$. The obtained results are showed in Fig. 2 in terms of radial temperature and velocity distributions at different sections of the duct. In second time, calculations are carried out for the same parameters but with various inclination angle and various water vapor molar fractions in order to investigate the effect of inclination angle on thermal and dynamic fields. The corresponding results are shown in Fig. 3.

The radial evolution of the temperature and of the axial velocity at different locations $(\mathrm{x}=0.01,0.1,0.4$, 0.7, 0.9 and $1.0 \mathrm{~m}$ ) are showed in Fig. 2. The calculations have been done either by including two components of the radiative flux vector or for non radiating medium (pure mixed convection) for $X_{\mathrm{H}_{2} \mathrm{O}}=0.6$ and $\theta=60^{\circ}$. The effect of the optical thickness on the thermal and on dynamic fields is showed in Fig. 2 for optically thin medium $(\mathrm{D}=0.03 \mathrm{~m})$ and for thick medium $(\mathrm{D}=0.1 \mathrm{~m})$. For comparison purpose of radiative heat transfer magnitude in these two cases, computations have been done for the same velocity profile at the entrance section $(\mathrm{x}=0)$.

The radial temperature evolutions of mixture, for $\mathrm{D}=0.03$ and $\mathrm{D}=0.1 \mathrm{~m}$ with taking into account thermal radiation and without thermal radiation, are plotted in Fig. 2a and b.

When radiative heat transfer is considered, the temperature values increase. The evolution of the temperature profile towards the wall is obviously faster with radiation than without radiation. The effect of thermal radiation magnitude is more pronounced in the center region of thick medium than for the thin one. In instance, in the center region at the section $\mathrm{x}=9 * \mathrm{D}$ the temperature is equal to $518 \mathrm{~K}$ for $\mathrm{D}=0.1 \mathrm{~m}$ while it is about $486 \mathrm{~K}$ for $\mathrm{D}=0.03 \mathrm{~m}$ when accounting for thermal radiation. In fact, radiative transfer from the wall acts at distance and leads to a more pronounced heating in central region of duct especially for thick medium.

The radial evolutions of the velocity are illustrated in Fig. 2c and d. A gradually distortion of the initial parabolic velocity profile is clearly observed, especially for an optically thick medium, due to the buoyancy forces.

The thermal radiation effects on the velocity fields are due to the variations of $\rho$ and $\mu$ with temperature. The radiative heat transfer effect is significant when the gas moves forwards; it reduces the velocity near the wall and increases it near the center region of the duct.

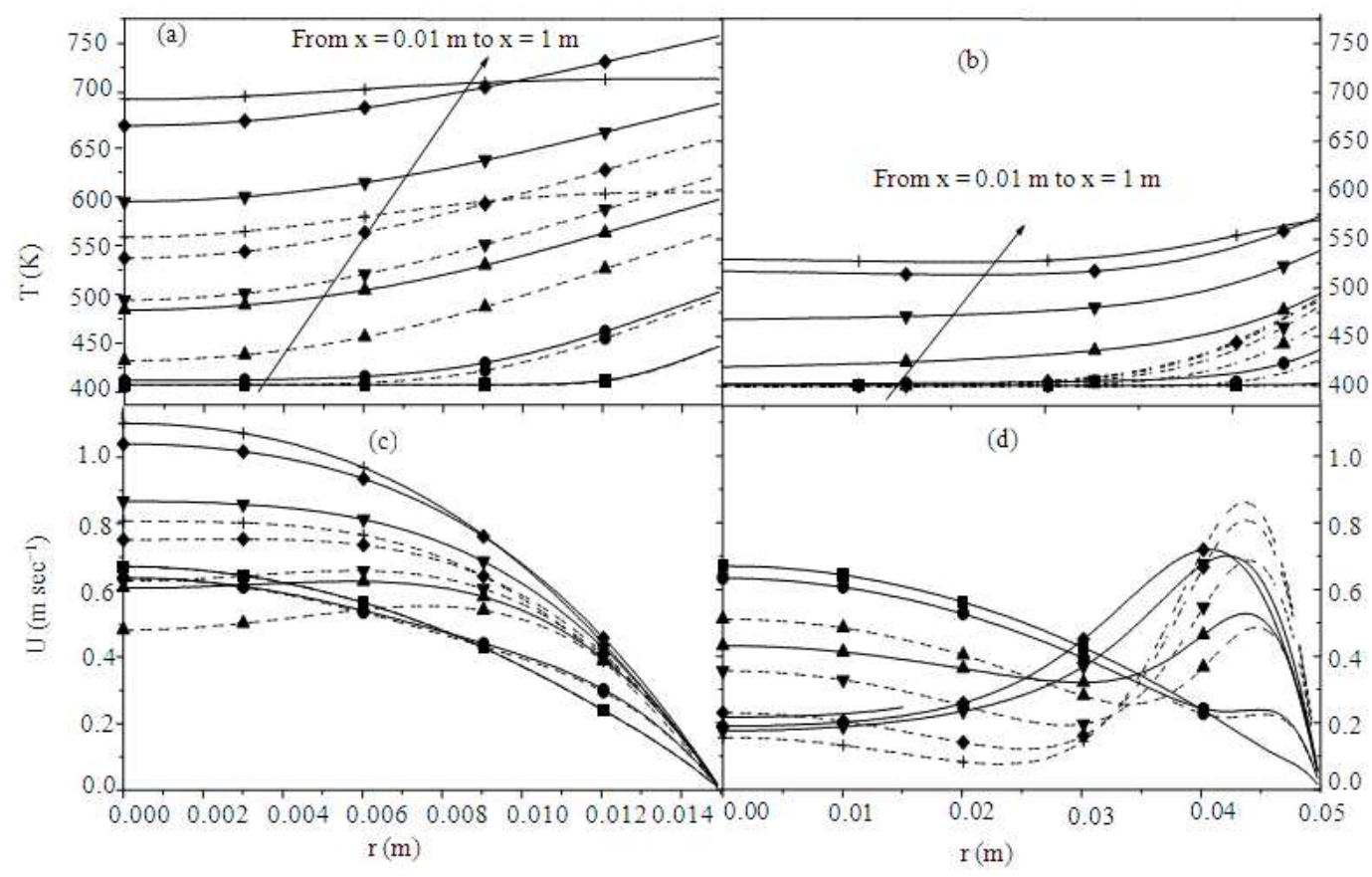

Fig. 2: Radial evolution of main temperature ( $\mathrm{a}$ and $\mathrm{b}$ ) and axial velocity (c and d) with $\mathrm{D}=0.03 \mathrm{~m}$ and $\mathrm{D}=0.1 \mathrm{~m}$ for different locations $(\boldsymbol{\nabla}): \mathrm{x}=0.01 \mathrm{~m},(\bullet): \mathrm{x}=0.1 \mathrm{~m},(\boldsymbol{\Delta}): \mathrm{x}=0.4 \mathrm{~m},(\boldsymbol{\nabla}): \mathrm{x}=0.7 \mathrm{~m},(\diamond): \mathrm{x}=0.9 \mathrm{~m}$ and $(+): x=1.0 \mathrm{~m} . \mathrm{T}_{0}=400 \mathrm{~K}, \mathrm{q}_{\mathrm{w}}=400 \mathrm{~W} \cdot \mathrm{m}^{-2}$ Solid line: Combined radiation and mixed convection Dashed line: Mixed convection without radiation 


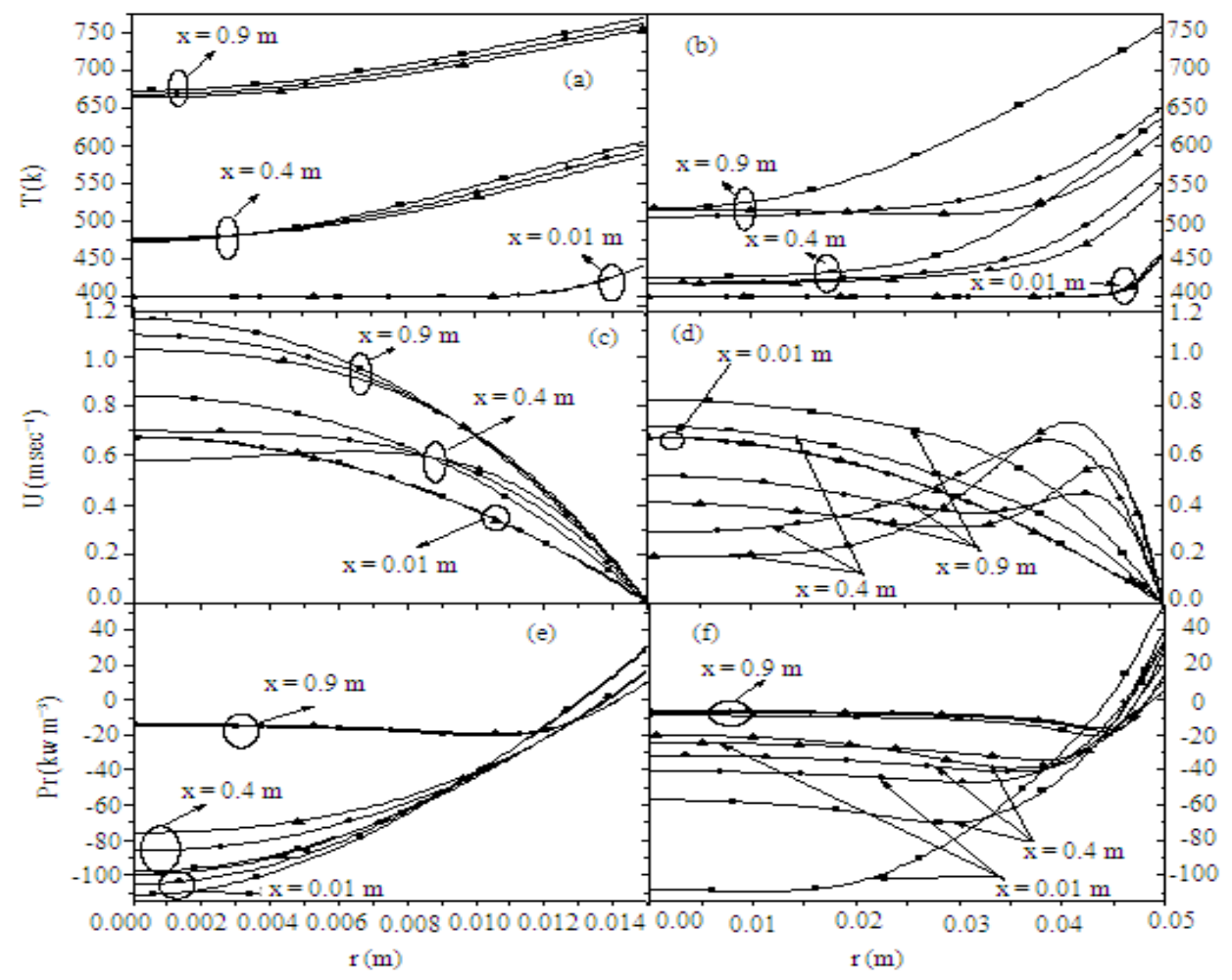

Fig. 3: Radial evolution of temperature ( $a$ and $b$ ), axial velocity ( $c$ and d) and volumetric radiative power dissipated in the medium (e and f) for $\mathrm{D}=0.03 \mathrm{~m}$ and $\mathrm{D}=0.1 \mathrm{~m}$ for different locations $\mathrm{x}=0.01,0.4,0.9 \mathrm{~m}$ and for $(\boldsymbol{\bullet}): \theta=0^{\circ},(\bullet): \theta=30^{\circ},(\boldsymbol{\Lambda}): \theta=90^{\circ} . \mathrm{T}_{0}=400 \mathrm{~K}$, Reynolds $=1500, \mathrm{q}_{\mathrm{w}}=400 \mathrm{~W} \mathrm{~m}^{-2}$

This behavior shows that radiation tends to reduce buoyancy effects as pointed out by Yang ${ }^{[23,24]}$ for gray media and Sediki et al. $^{[3]}$ for non-gray radiative media. So radiation strongly modifies the temperature distributions and affects the main temperature.

Effects of inclination angle on velocity, temperature and radiative power distributions: The main purpose of the calculus presented to point out the effect of different cylindrical orientations on the developing of thermal and dynamic fields. Figure 3 shows, the radial evolution of the temperature, the velocity and the radiative power profiles for various inclination angle and at different sections $(\mathrm{x}=0.01,0.4,0.9$ and $1.0 \mathrm{~m})$. Results for both cases (thin and thick medium) are also presented in the same Fig. 3.

Effect of inclination angle on the temperature distribution: The radial temperature distributions for different sections $(\mathrm{x}=0.01,0.4,0.9$ and $1 \mathrm{~m})$ and for various inclination angle $\theta=0^{\circ}$ (horizontal), $30^{\circ}$ and $90^{\circ}$ (vertical) are plotted in Fig. $3 \mathrm{a}$ and $3 \mathrm{~b}$. In the entrance region of the duct $(x=0.01 \mathrm{~m})$, the temperature fields stay uniform. The effect of inclination angle can not be detected in this region. In fact, the temperature is almost equal to the inlet temperature and the velocity field still parabolic. The influence of the duct inclination angle on the thermal field was better depicted for thick medium.

Hence, the temperature rate flow can not be established as long as the heat flux is imposed at the duct wall. In the section $x=0.4 \mathrm{~m}$, the velocity near the wall is greater for vertical duct than for horizontal one and the fluid seems to be accelerated near the wall for cylindrical inclined case.

Simultaneously the temperature near the wall is higher for horizontal duct than for vertical one. Contrary, near the center region, the fluid seems to be decelerated for vertical tube so the temperature is relatively high. Due to the temperature dependent density the fluid has low velocity for high temperature and vice-versa; this is mainly due to the effect of radiation on the velocity distributions.

Effect of inclination angle on the velocity: Figure 3c and $d$ show the effect of duct inclination angle on the 
velocity profiles along the radial direction. In the entrance region of the duct $(x=0.01 \mathrm{~m})$, the effects of inclination angle can not be noticeable and the velocity profile remains parabolic. When the fluid is flowing away in the duct (from $\mathrm{x}=0.4 \mathrm{~m}$ ), the velocity profile is gradually distorted and we can discern the inclination angle effects. For the horizontal orientation case, the fluid is more pushed than in vertical one. This is due to opposing buoyancy forces which is maximal in the vertical case and may gives rise to flow reversal. As the fluid is going on, the velocity shape tends in vain to become parabolic. The strongest inclination influence takes place for $\theta>30^{\circ}$. Near the center, the velocity decreases when $\theta$ increases, while near the wall, the velocity increases when $\theta$ decreases.

Effect of inclination angle on radiative power: The developing radiative power profiles at different sections are illustrated in Fig. 3d and e. It is clearly shown that around the duct center the radiative power values are negatives; this is a result of the transferred radiative flux from the hot wall surface to the cold fluid. In fact, the radiative energy travels into the deep central core from heated region to the central region. Furthermore, the radiative power is less important for the horizontal duct $\left(\theta=0^{\circ}\right)$ than for the vertical duct. This is due to weak temperature difference between the wall and the centre.

Indeed, for one section, the fluid near the wall is heated not only by conduction from the wall but also by convection from upstream heated section.

The difference between the main temperature and the wall temperature decreases for upper sections. The main temperature tends to the wall temperature then declines after a maximum value at $\mathrm{x}=0.9 \mathrm{~m}$; because beyond this region the heat flux is maintained equal to zero at the wall. Therefore, the radiative power profile is flat due to the homogenization of the temperature in the fluid; thus, the effect of inclination angle is more pronounced at the central region than near the wall.

Bulk and wall temperatures distributions: Figure 4 shows the axial evolution of bulk temperature for different inclination angles $\left(\theta=0^{\circ}, 30^{\circ}\right.$ and $\left.90^{\circ}\right)$ and for different water vapor molar fractions $\left(\mathrm{X}_{\mathrm{H}_{2} \mathrm{O}}=0.6,0.2\right.$ and 1.0) computed with and without taking into account thermal radiation. The inlet temperature of gas is equal to $400 \mathrm{~K}$ and the uniform heat flux at the wall is equal to $400 \mathrm{~W} \mathrm{~m}^{-2}$. The case of a thick medium $(\mathrm{D}=0.1 \mathrm{~m})$ is considered and a value of Reynolds number is fixed at 1500 .
Figure 4 shows that for pure convection, effects of water vapor molar fraction and inclination angle on bulk temperature are not significant. However, when accounting for radiative heat transfer, the bulk temperature increases from the inlet region of the duct and reach a maximum value at the outlet region. It is observed that for the same inclination angle the bulk temperature is high for high $\mathrm{X}_{\mathrm{H}_{2} \mathrm{O}}$ value. In instance, at the outlet section and for $\theta=30^{\circ}, \mathrm{T}_{\mathrm{b}}$ is about $650 \mathrm{~K}$ when $\mathrm{X}_{\mathrm{H}_{2} \mathrm{O}}=1.0$, while $\mathrm{T}_{\mathrm{b}}$ is about $500 \mathrm{~K}$ when $\mathrm{X}_{\mathrm{H}_{2} \mathrm{O}}=0.2$. This is a consequence of direct effect of the radiative transfer on temperature distribution and its indirect effect on velocity. In fact, the flow is decelerated when the density increases. Moreover, when accounting for thermal radiation, the disparity between these bulk temperatures is greater than for the case without radiation. Obviously, radiative transfer accelerates the thermal development. Also, for the same water vapor molar fraction, the bulk temperature increases with the decrease of inclination angle, for example, at the section $\mathrm{x}=0.8 \mathrm{~m}$ and for $\mathrm{X}_{\mathrm{H}_{2} \mathrm{O}}=1.0$, $\mathrm{T}_{\mathrm{b}}=675 \mathrm{~K}$ with $\theta=0^{\circ}$ and $\mathrm{T}_{\mathrm{b}}=575 \mathrm{~K}$ with $\theta=90^{\circ}$.

Figure 5 show the axial evolution of wall temperature for different molar fractions of water vapor $\left(\mathrm{X}_{\mathrm{H} 2 \mathrm{O}}=0.2,0.6\right.$ and 1.0) and for different inclination angles of duct $\left(\theta=0^{\circ}, 30^{\circ}\right.$ and $\left.90^{\circ}\right)$ computed with and without taking into account the thermal radiation (respectively shown in Fig. 5a and b). The same trends are observed for heat transfer with convection alone and with combined convection and radiation heat transfer.

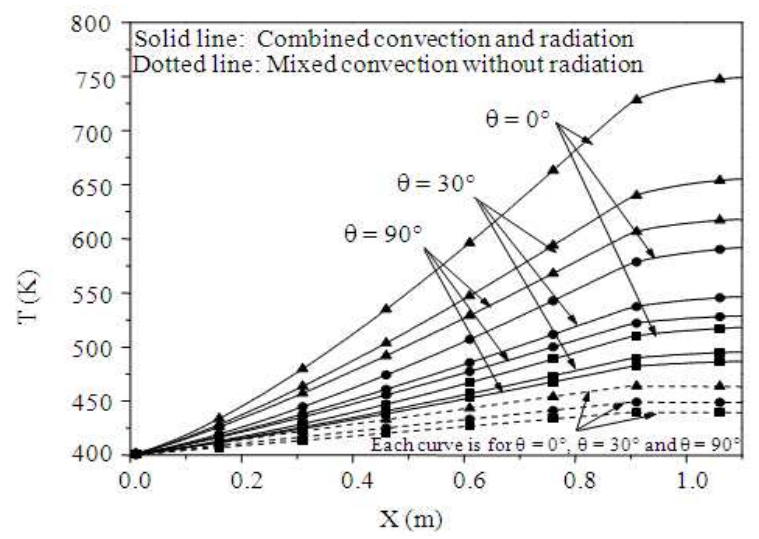

Fig. 4: Evolution of the bulk temperature for different molar fractions of gas with $\mathrm{X}_{\mathrm{H} 2 \mathrm{O}}=0.2(\boldsymbol{\square})$; $\mathrm{X}_{\mathrm{H}_{2} \mathrm{O}}=0.6(\bullet) ; \mathrm{X}_{\mathrm{H}_{2} \mathrm{O}}=1.0(\boldsymbol{\Delta})$ and for different inclination angle of duct. $\mathrm{D}=0.1 \mathrm{~m}, \mathrm{~T}_{0}=400 \mathrm{~K}$, Reynolds $=1500, \mathrm{q}_{\mathrm{w}}=400 \mathrm{~W} \mathrm{~m}^{-2}$ 
Am. J. Engg. \& Applied Sci., 2 (4): 590-602, 2009

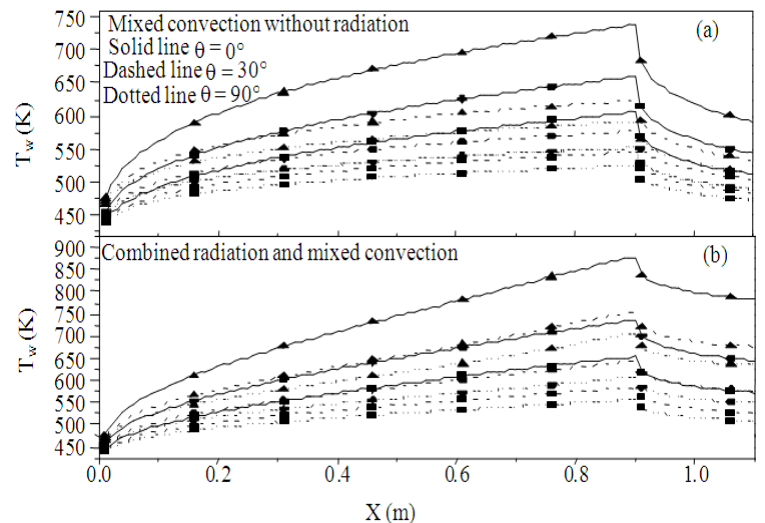

Fig. 5: Evolution of the wall temperature for different molar fractions of gas with $\mathrm{X}_{\mathrm{H}_{2} \mathrm{O}}=0.2(\mathbf{-})$; $\mathrm{X}_{\mathrm{H}_{2} \mathrm{O}}=0.6(\bullet) ; \mathrm{X}_{\mathrm{H}_{2} \mathrm{O}}=1.0(\boldsymbol{\Delta})$ and different inclination angle of duct. $\mathrm{D}=0.1 \mathrm{~m}, \mathrm{~T}_{0}=400$ $\mathrm{K}$, Reynolds $=1500, \mathrm{q}_{\mathrm{w}}=400 \mathrm{~W} \mathrm{~m}^{-2}$

The obtained results let see the constantly increase on the values of wall temperature from the inlet region of the duct to reach a maximum value at the location $\mathrm{x}=0.9 \mathrm{~m}$ beyond which temperature values begin to decrease since up to the location $\mathrm{x}=0.9 \mathrm{~m}$ the heat flux value is zero. It is also shown that radiation increases the wall temperature (at $\mathrm{x}=0.9 \mathrm{~m}, \theta=0^{\circ}$ and $\mathrm{X}_{\mathrm{H}_{2} \mathrm{O}}=1.0$ Fig. $5 \mathrm{a} \mathrm{T}_{\mathrm{w}}=875 \mathrm{~K} ; 5 \mathrm{~b} \mathrm{~T} \mathrm{w}_{\mathrm{w}}=740 \mathrm{~K}$. The wall temperature difference between both cases (combined modes and pure mixed convection) is about $135 \mathrm{~K}$ for the case of $\mathrm{X}_{\mathrm{H}_{2} \mathrm{O}}=1.0$ and $\theta=0^{\circ}$ whereas it decreases about $17 \%$ for $\mathrm{X}_{\mathrm{H}_{2} \mathrm{O}}=1.0$ and $\theta=30^{\circ}$. Therefore the inclination angle of the duct has not significant effect on the thermal radiation heat transfer. Moreover, the developing of thermal boundary layer is faster for gases having high water vapor molar fraction than for low fraction.

Nusselt number evolution: Figure 6 shows the effect of thermal radiation of water vapor molar fractions and the effect of duct inclination angle on the Nusselt number $\left(\mathrm{N}_{\mathrm{u}}\right)$ distribution. Results are obtained when radiation is accounting for and for duct diameter equal to $0.1 \mathrm{~m}$ (thick medium). Nusselt number has high values at the entrance of the duct similar to the flat plate then it decreases monotonically and tends to an asymptotic value which highly depends on water vapor molar fraction due to the nonlinearity of the diverse magnitude of thermal radiation effect.

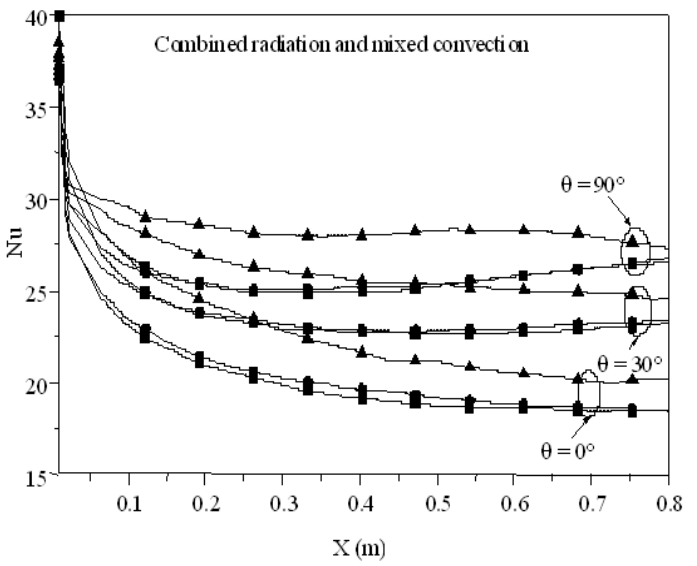

Fig. 6: Evolution of Nusselt number for different molar fractions of gas with $\mathrm{X}_{\mathrm{H}_{2} \mathrm{O}}=0.2(\boldsymbol{\bullet}) ; \mathrm{X}_{\mathrm{H}_{2} \mathrm{O}}=0.6$ $(\bullet) ; \mathrm{X}_{\mathrm{H}_{2} \mathrm{O}}=1.0(\boldsymbol{\Delta})$ and for different inclination angle of duct. $\mathrm{D}=0.1 \mathrm{~m}, \quad \mathrm{~T}_{0}=400 \mathrm{~K}$, Reynolds $=1500, \mathrm{q}_{\mathrm{w}}=400 \mathrm{~W} \mathrm{~m}^{-2}$

The effect of duct inclination angle on Nusselt number is also observed. Nusselt number increases with the increase of the inclination angle. So, for horizontal duct the values of Nusselt number are lower than for the vertical case due to the buoyancy forces effect. Thus, conduction heat transfer is more significant for horizontal cylinder and its effect is reduced as duct orientation moves to the vertical position. This result is obviously due to high temperature in horizontal tube as discussed before. In instance, for water vapor molar fraction equal to 0.2 the asymptotic value of Nusselt number is about 19 for $\theta=0^{\circ}$ and about 27 for $\theta=90^{\circ}$. Nevertheless, for the same inclination angle, the Nusselt number increases with $\mathrm{X}_{\mathrm{H}_{2} \mathrm{O}}$, the higher value of $\mathrm{N}_{\mathrm{u}}$ is reached when the water vapor molar fraction of the gas is equal to 1.0. This behavior can be explained by to the thermal radiation effect.

The local Nusselt number corresponding to coupled convection and radiation as well as mixed convection alone are illustrated in Fig. 7. Results are obtained for gases with different water vapor molar fractions. The inlet temperature of gas is equal to $400 \mathrm{~K}$ and the uniform heat flux at the wall is equal to $400 \mathrm{~W} \mathrm{~m}^{-2}$. The case of a thick medium $(\mathrm{D}=0.1 \mathrm{~m})$ is considered and a value of Reynolds number is fixed at 1500 .

Nusselt number profiles are given for two optical thicknesses of duct $(\mathrm{D}=0.03$ and $\mathrm{D}=0.1 \mathrm{~m})$ with a fixed position of an inclined duct $\left(\theta=30^{\circ}\right)$.

The total Nusselt number is higher for optically thick medium $(\mathrm{D}=0.1 \mathrm{~m})$ than for optically thin one $(\mathrm{D}=0.03 \mathrm{~m})$. This is due to the thermal radiation 
contributions in heat transfer. Indeed, the effect of radiation rises with the optical thickness and the conduction mode is predominant for weak diameter $(\mathrm{D}=0.03 \mathrm{~m})$. It is clearly shown that for thin medium Nusselt number values are slightly affected by thermal radiation whereas effect of thermal radiation is well pronounced for the case of thick medium. For thin medium and when radiation is not considered, the classical result of the value of Nusselt number equal to 4.66 for laminar forced convection flow inside circular duct with imposed uniform heat flux was found.

Discussion of numerical and experimental data: Here, the numerical solution method for combined convection and thermal radiation presented above is used to predict the bulk temperature and the Nusselt Number for physical and geometrical conditions corresponding to the experiments conducted by Mohammed and Salman ${ }^{[16]}$. Numerical calculations are performed with the measured surface temperatures and uniform wall heat flux ${ }^{[16]}$. According to which boundary conditions used, the dynamic, the thermal and the radiative fields are computed. Then the bulk temperature and the Nusselt are deduced from the numerical results by using Eq. 9 and 11 respectively where experimental data of ${ }^{[16]}$ are input as boundary conditions.

Experimental study of Mohammed and Salman is conducted in order to study the effect of cylinder inclination angle and wall heat flux values on the laminar air flow under mixed convection heat transfer inside uniformly heated circular tube.

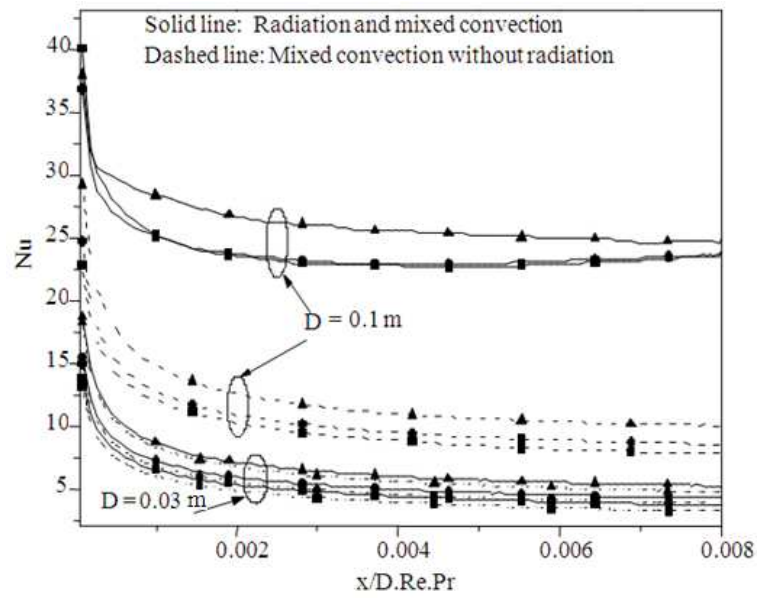

Fig. 7: Evolution of Nusselt number for two diameters and different molar fractions of gas $\left(\mathrm{X}_{\mathrm{H}_{2} \mathrm{O}}=0.2\right.$ $\left.(\boldsymbol{\bullet}) ; \mathrm{X}_{\mathrm{H}_{2} \mathrm{O}}=0.6(\bullet) ; \mathrm{X}_{\mathrm{H}_{2} \mathrm{O}}=1.0(\boldsymbol{\Delta})\right) . \mathrm{T}_{0}=400 \mathrm{~K}$, Reynolds $=1500, \mathrm{q}_{\mathrm{w}}=400 \mathrm{Wm}^{-2}$
The geometrical cylindrical parameters selected from the experimental series are defined by $\mathrm{L}=0.9 \mathrm{~m}$, $\mathrm{D}=0.03 \mathrm{~m}$ and $\theta=30^{\circ}$ and Reynolds $=400$. The authors have computed the local bulk air temperature by fitting straight line-interpolation between the measured inlet and outlet bulk air temperatures with two thermocouples placed in these locations. The cylinder surface temperatures were measured by 35 thermocouples fixed along the cylinder.

The average values of Nusselt number are evaluated with the constant wall heat flux, the average surface temperature and the average bulk air temperature. Moreover, Mohammed and Salman ${ }^{[16]}$ have given, in instance a correlation of the average Nusselt values in empirical equation as follow:

$$
\overline{\mathrm{N}} \mathrm{u}=3.25\left(\frac{\overline{\mathrm{R}} \mathrm{a}}{\overline{\mathrm{Re}}}\right)^{0.187} \text { for } \theta=30^{\circ}
$$

A series of numerical calculations have been done in the same conditions of this experience, but with taking into account the thermal radiation mode due to the presence of radiative species (water vapor in air). So, the fluid bulk temperature was determined numerically from measured surface temperature values taken as a data from experimental study of Mohammed and Salman ${ }^{[16]}$. Nusselt numbers were computed one time by using the constant uniform wall heat flux value $\left(q_{w}=396 \mathrm{~W} \mathrm{~m}{ }^{-2}\right)$ and other one with surface temperature values deduced from ${ }^{[16]}$.

Numerical results are plotted in Fig. 8. Figure 8a and $8 \mathrm{~b}$ showed numerical and experimental results in terms of bulk, surface temperatures and Nusselt number variations. In Fig. 8a numerical results of bulk temperature calculated referring to surface temperature profile find in the literature is shown. Evolution of Nusselt number using once the condition at the wall subjected to uniform heat flux and using the surface temperature values is presented in Fig. 8b. For comparison purpose, Nusselt number profile obtained by Shah and London ${ }^{[34]}$ is added in Fig. 8b, in order to compare with experimental investigation of Mohammed and Salman ${ }^{[16]}$.

The experimental Nusselt number values obtained by Mohammed and Salman are far from the calculated values either by using the condition of uniform heat flux at the wall or by using the experimental values of the surface temperature. Moreover, Nusselt numbers obtained by Shah and London ${ }^{[34]}$ are almost of the same order than numerical results.

Firstly, the parabolic surface temperature profile creases to a maximum value and then decreases in accordance with heat losses inside the duct. In fact, 
Am. J. Engg. \& Applied Sci., 2 (4): 590-602, 2009

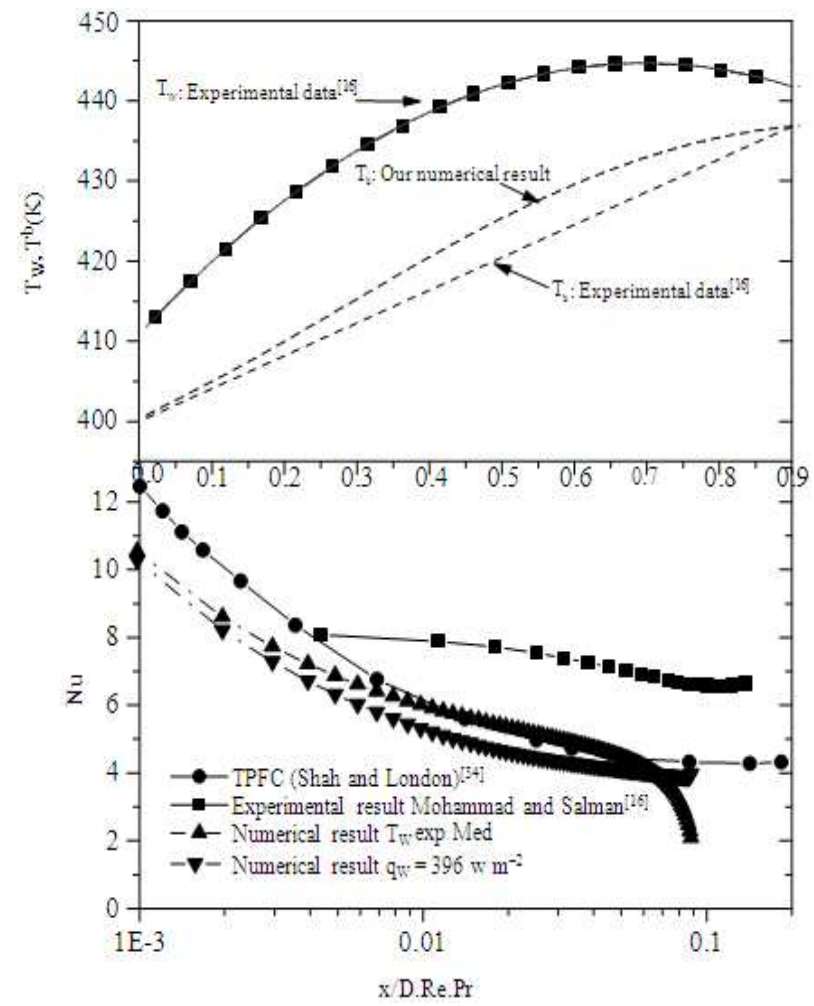

Fig. 8: Comparison between numerical calculations and experimental results of surface temperature and Bulk temperature (a) and Nusselt number distributions (b) for laminar flow of steam. With $\operatorname{Re}=400$ and $\theta=30^{\circ}$, experimental results of Mohammed and Salman ${ }^{[16]}(\boldsymbol{\bullet}),(\bullet)$ TPFC, (--) numerical results for experimental values of $T_{w}$ find by Mohammed and Salman ${ }^{[16]}(\boldsymbol{\nabla})$ for in uniform wall heat flux $\mathrm{q}_{\mathrm{w}}=396 \mathrm{~W} \mathrm{~m}^{-2}(\boldsymbol{\Lambda})$

such surface temperature profile corresponds to variable wall heat flux instead of a constant one submitted on the tube (from section $=0.0$ to section $=0.9 \mathrm{~m}$ ).

Thus, the wall heat flux was in reality variable and can not be considered constant. Furthermore, the dimensionless section $\mathrm{x} / \mathrm{D}$. Re. $\operatorname{Pr}$ (with $\operatorname{Pr}=\mu \cdot \mathrm{C}_{\mathrm{p}} / \lambda$ ) is function of thermophysical properties which depend on the main temperature of the fluid. If the measured wall duct surface temperature values are adopted when computing numerically Nusselt number, a significant differences with experimental results are shown in Fig. 8b. These differences are mainly caused by the bulk temperature. In fact, the bulk temperature profile obtained from the parabolic surface temperature is not linear as considered in literature ${ }^{[16]}$. $\operatorname{In}^{[16]}$, the bulk temperature evolution is determined from only two points in the beginning and in the end of the cylinder that is subjected to constant heat flux which is in reality variable.

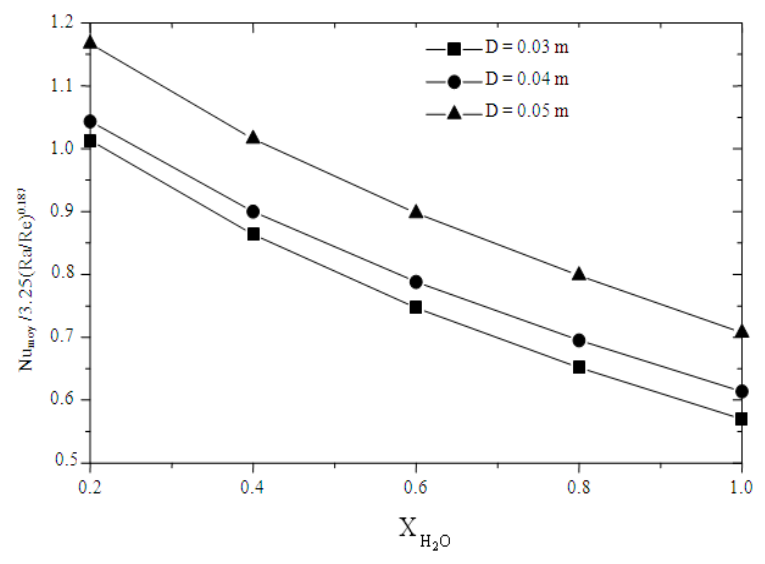

Fig. 9: Evolution of numerical average Nusselt number on experimental correlation values for different molar fractions of gas and three diameters $(\mathrm{D}=$ $0.03 \mathrm{~m}(\bullet), \mathrm{D}=0.04 \mathrm{~m}(\bullet), \mathrm{D}=0.05 \mathrm{~m}(\boldsymbol{\Delta}))$. $\mathrm{T}_{0}=400 \mathrm{~K}$, Reynolds $=400, \mathrm{q}_{\mathrm{w}}=396 \mathrm{~W} \cdot \mathrm{m}^{-2}$

Lastly, experimental Nusselt numbers are based on a constant value of flux, a linear bulk temperature, a constant thermal conductivity and on interpolated mean temperature evolution deduced from two positions. All these hypotheses explain the difference between numerical and experimental results.

Numerical investigations of the average Nusselt numbers for different molar fractions of gas and for various diameters $(0.03 ; 0.04$ and $0.05 \mathrm{~m})$ are plotted in Fig. 9 as well as and experimental results. The numerical average Nusselt numbers are underestimated compared to those given by Mohammed and Salman ${ }^{[16]}$. For the cases corresponding to $\left(\mathrm{X}_{\mathrm{H} 2 \mathrm{O}}\right.$ different to 0.2 and $\mathrm{D}$ different to $0.03 \mathrm{~m}$ ), a noticeable discrepancy is observed between the numerical values of Nusselt and its experimental correlation. This divergence is due to the presence of radiative molecular species which increases with the diameter of the duct.

For large application taking into account the nongray radiative behavior of mixtures, correlation formula between average Nusselt number and optical thickness of the media appear as a natural extension of this investigation.

\section{CONCLUSION}

In this study, we have presented a comprehensive treatment of combined radiation and mixed convection for a participating fluid flowing inside an inclined duct. However, numerical investigation was performed to study the effect of duct inclination angle on the heat transfer characteristics with various gas compositions. 
The general trends and effects due to thermal radiation, such as the enhancement of the total heat transfer, the distortion of velocity profiles for heated flows, the reduction of buoyancy forces effects, are recovered when the physical parameters are changed. One comparison with experimental results is presented. From the investigation the following conclusions can be drawn:

The velocity of fluid decreases with the increase of the inclination angle of the duct. At the entrance region, the inclination has no effect on the velocity profiles. As the fluid moves on the velocity profile is gradually distorted and the inclination effects are discerned.

The thermal radiation effects on thermal and dynamic fields are clearly observed and cannot be ignored in such medium containing radiative participating gaseous mixtures.

Thermal radiation, when it is taken into account, enhances heat transfer and speeds up the evolution of bulk temperature towards wall temperature.

The heat transfer depends upon the geometry of the duct and up on the composition of the gas flowing inside the duct. So, Nusselt number increases with the inclination angle, the diameter of the duct and the molar fraction of the radiating gaseous mixtures.

Finally, the proposed correlation of the average Nusselt number obtained by experimental investigation was compared to the numerical results in the same conditions. It is important to highlight the dependence of the average Nusselt numbers with the optical thickness of the gaseous mixtures.

\section{REFERENCES}

1. Viskanta, R., 1998.Overview of convection and radiation in high temperature gas flows. Int. J. Eng. Sci., 36: 1677-1699. DOI: 10.1016/S00207225(98)00053-6

2. Sediki, E., A. Soufiani and M.S. Sifaoui, 2002. Spectrally correlated radiation and laminar forced convection in the entrance region of circular duct. Int. J. Heat Mass Trans., 45: 5069-5081. DOI: 10.1016/S0016-9310(02)00205-3

3. Sediki, E., A. Soufiani and M.S. Sifaoui, 2003. Combined gas radiation and laminar mixed convection in vertical circular tubes. Int. J. Heat Fluid Flow, 24: 736-746. DOI: 10.1016/S0142727X(03)00005-5

4. Jackson, J.D., M.A. Cotton and B.P. Axcell, 1989. Studies of mixed convection in a vertical tube. Int. J. Heat Fluid Flow, 10: 2-15. DOI: 10.1016/0142727 X(89)90049-0
5. Jeng, Y.N., J.L. Chen and W. Aung, 1992. On the reynolds-number independence of mixed convection in a vertical channel subjected to asymmetric wall temperatures with and without flow reversal. Int. J. Heat Fluid Flow, 13: 329-339. DOI: 10.1016/0142-727X(92)90003-R

6. Hottel, H.C. and A.F. Sarofim, 1967. Radiative Transfer. 2nd Edn., McGraw-Hill, New York, pp: 520. http://books.google.com.pk/books?id=C7U8AAA AIAAJ\&q=Radiative+Transfer \&dq=Radiative+Tra nsfer\&pgis $=1$

7. Modest, M.F.,1993. Radiative Heat Transfer. 2nd Edn., McGraw Hill, New York, ISBN: 0-12503163-7

8. Mohammed, H.A. and Y.K. Salman, 2007. The effects of different entrance sections lengths and heating on free and forced convective heat transfer inside a horizontal circular tube. Int. Commun. Heat Mass Transfer, 34: 769-784. DOI: 10.1016/j.icheatmasstransfer. 2007.03.005

9. Mohammed, H.A. and Y.K. Salman, 2007. Free and forced convection heat transfer in the thermal entry region for laminar flow inside a circular cylinder horizontally oriented. Energy Convers. Manage., 48: 2185-2195. DOI: 10.1016/j.enconman.2006.12.016

10. Mohammed, H.A. and Y.K. Salman, 2007. Experimental investigation of mixed convection heat transfer for thermally developing flow in a horizontal circular cylinder. Applied Thermal Eng., 27: 1522-1533. DOI: 10.1016/j.applthermaleng.2006.09.023

11. Mohammed, H.A., 2009. The effect of different inlet geometries on laminar flow combined convection heat transfer inside a horizontal circular pipe. Applied Thermal Eng., 29: 581-590. DOI: 10.1016/j.applthermaleng.2008.03.024

12. Mohammed, H.A. and Y.K. Salman, 2007. Heat transfer by natural convection from a uniformly heated vertical circular pipe with different entry restriction configurations. Energy Convers. Manage., 48: 2244-2253. DOI: 10.1016/j.enconman.2006.12.005

13. Mohammed, H.A. and Y.K. Salman, 2007. Combined natural and forced convection heat transfer for assisting thermally developing flow in a uniformly heated vertical circular cylinder. Int. Commun. Heat Mass Transfer, 34: 474-491. DOI: 10.1016/j.icheatmasstransfer.2007.01.001

14. Mohammed, H.A. and Y.K. Salman, 2007. Free convective heat transfer from a constant heat flux vertical circular tube with different entrance restrictions length. Energy Convers. Manage., 48: 2233-2243. DOI: 10.1016/j.enconman.2006.12.024 
15. Mohammed, H.A., 2008. Laminar mixed convection heat transfer in a vertical circular tube under buoyancy-assisted and opposed flows. Energy Convers. Manage., 49: 2006-2015. DOI:10.1016/j.enconman.2008.02.009

16. Mohammed, H.A. and Y.K. Salman, 2007. Combined convection heat transfer for thermally developing aiding flow in an inclined circular cylinder with constant heat flux. Applied Thermal Eng., 27: 1236-1247. DOI: 10.1016/j.enconman.2008.02.009

17. Seo, T., D.A. Kaminski and M.K. Jensen, 1994. Combined convection and radiation in simultaneously developing flow and heat transfer with non gray gas mixtures. Num. Heat Trans., 26: 49-66. DOI: 10.1080/10407789408955980

18. Selçuk, N., A.B. Uygur, I. Ayranci and T. Tahran, 2005. Transient simulation of radiation flows. J. Quantitat. Spectroscopy Radiat. Trans., 93: 151-161. DOI: 10.1016/j.jqsrt.2004.08.034

19. Chiu, H.C. and W.M. Yan, 2008. Mixed convection heat transfer in inclined rectangular ducts with radiation effects. Int. J. Heat Mass Trans., 51: 1085-1094. DOI: 10.1016/j.ijheatmasstransfer. 2007.05.009

20. Greif, R., 1978. Laminar Convection with radiation experimental and theoretical results. Int. J. Heat Mass Trans., 21: 477-480. DOI: 10.1016/00169310(78)90081-9

21. Smith, T.F. and C.W. Clausen, 1978. Radiative and convective transfer for tube flow of a real gas. Proceeding of the International Conference on Heat Transfer, Aug. 7-11, Toronto, Canada, pp: 391-396. http://adsabs.harvard.edu/abs/1978iht.....3..391S

22. Kim, T.K. and T.F. Smith, 1985. Radiative and conductive transfer for of a real gas in a cylindrical enclosure with gray walls. Int. J. Heat Mass Trans., 28: 2269-2277. DOI: $10.1016 / 0016-$ 9310(85)90045-6

23. Yang, L.K., 1991. Combined mixed convection and radiation in a vertical pipe. Int. J. Heat Mass Trans., 18: 419-430. DOI: 10.1016/07351933(91)90058-C

24. Yang, L.K., 1992. Forced convection in a vertical pipe with combined buoyancy and radiation effects. Int. J. Heat Mass Trans., 19: 249-262. DOI: 10.1016/0735-1933(92)90036-H

25. Taine, J. and A. Soufiani, 1999. Gas IR radiative properties from spectroscopic data to approximate models. Adv. Heat Transfer, 33: 295-414. http://cat.inist.fr/?aModele $=$ afficheN\&cpsidt $=1721$ 794
26. Pierrot, L., PH. Rivière, A. Soufiani and J. Taine, 1999. A fictitious-gas-based absorption distribution function global model for radiative transfer in hot gases. J. Q. Spectroscopy Radiat. Trans., 62: 609-624. DOI: 10.1016/S0022-4073(98)00124-1

27. Touloukian, Y.S., 1970. Thermophysical Properties of Matter, IFI/Plenum, New York. ISBN: 0306670208.

28. Lakhal, W., S. Trabelsi, E. Sediki, A. Soufiani and M. Moussa, 2007. Computational spectrally correlated thermal radiation through gaseous mixture. Proceeding of AIP Conference, pp: 244-249. http://adsabs.harvard.edu/abs/2007AIPC..935..244 $\mathrm{L}$

29. Patankar, S.V. and D.B. Spalding, 1972. A calculation procedure for heat mass and momentum transfer in three dimensional parabolic flows. Int. J. Heat Mass Trans., 15: 1687-1806. DOI: 10.1016/0016-9310(72)90054-3

30. Bankston, C.A. and D.M. McEligot, 1970. Turbulent and laminar heat transfer to gases with varying properties in the entry region of circular ducts. Int. J. Heat Mass Trans., 13: 319-343. DOI: 10.1016/0016-9310(70)90110-9

31. Worsoe-Schmidt, P.M. and G. Leppert, 1965. Heat transfer and friction for laminar flow of gas in a circular tube at high heating rate. Int. J. Heat Mass Trans., 8: 1281-1301. DOI: 10.1016/00169310(65)90056-6

32. Aung, W. and G. Worku, 1986. Developing flow and flow reversal in a vertical channel with asymmetric wall temperatures. J. Heat Transfer, 108: 299-304.

http://cat.inist.fr/?aModele $=$ afficheN\&cpsidt $=7912$ 172

33. Aung, W. and G. Worku, 1986. Theory of fully developed, combined convection including flow reversal. J. Heat Transfer, 108: 485-488. http://cat.inist.fr/?aModele $=$ afficheN\&cpsidt $=7912$ 184

34. Shah, R.K. and A.L. London, 1978. Laminar flow forced convection in ducts: Supplement 1 to Advances in heat transfer. Academic Press, ISBN: 0120200511, pp: 477. 